\title{
AVALIAÇÃO DA QUALIDADE FÍSICA, FISIOLÓGICA E SANITÁRIA DE SEMENTES DE MILHO DURANTE O BENEFICIAMENTO ${ }^{1}$
}

\author{
SIMONE APARECIDA FESSEL ${ }^{2}$; RUBENS SADER ${ }^{3} ;$ RINALDO CESAR DE PAULA $^{4} ;$ JULIANA ALTAFIN GALLI $^{5}$
}

\begin{abstract}
RESUMO - A pesquisa teve por objetivo avaliar a qualidade física, fisiológica e sanitária de sementes de milho durante as etapas de beneficiamento. Foram utilizadas 16 amostras de um lote de sementes do híbrido D766 safra 2001/2002 , classificado em sementes chatas médias curtas e redondas curtas, de peneiras 20 e 22. As amostras foram obtidas durante as fases do beneficiamento de recepção, pré-limpeza, pós-mesa de gravidade, pós-classificador e pós-ensaque. As sementes foram avaliadas quanto ao teor de água, germinação, primeira contagem da germinação, índice de velocidade de germinação, envelhecimento acelerado, teste de frio, condutividade elétrica, emergência das plântulas em campo, sanidade, através do teste de papel filtro e dano mecânico. Para análise estatística utilizou-se o delineamento inteiramente casualizado, com quatro repetições, e a comparação das médias foi feita usando-se o programa SAEG, teste de comparações múltiplas de Scott-Knott a 5\% de probabilidade. Os resultados deste trabalho sugeriram que os danos mecânicos podem ocorrer em cada fase do processamento e são cumulativos e o beneficiamento de sementes de milho pode aprimorar a qualidade de sementes de um lote em termos de germinação, vigor e sanidade.

Termos para indexação: Zea mays L., germinação, vigor, processamento, injúria mecânica.
\end{abstract}

\section{QUALITY EVALUATION OF CORN SEEDS DURING CONDITIONING}

\begin{abstract}
The objective of this research was to evaluate the physical, physiological and sanitary quality of corn seeds during conditioning. Sixteen samples of a corn seed lot hybrid D766 produced in 2001/2002 was used, classified as medium short flat and short round, from the screens 20 and 22. The seed samples were obtained during the following conditioning steps: reception, pre-cleaning, post-gravity separator, post-grader and after bagging. The seeds were evaluated for the moisture content, standard germination, first germination count, speed of germination index, accelerated aging, cold test, electrical conductivity, seedling field emergence, health test through the filter paper and injury test. The experimental design used was completely randomized with 4 replications and the mean comparison was made through the Scott-Knott multiple path comparison test at $5 \%$ level of probability. According to the obtained results it was concluded that mechanical injury can occur at any stage of seed conditioning and is cumulative and the corn seed conditioning may improve the seed lot quality in for germination, vigor and health.

Index terms: Zea mays L. germination, vigor, processing, mechanical injury.
\end{abstract}

\section{INTRODUÇÃO}

O beneficiamento de sementes constitui-se em uma etapa essencial na produção de sementes de alta qualidade, visto

\footnotetext{
1 Aceito para publicação em 20/11/2003.

2,5 Eng ${ }^{\text {os }} \mathrm{Agr}^{\text {os }}$, estudantes de Pós-Graduação em Agronomia, FCAV/UNESP - Câmpus de Jaboticabal, SP, 14884-900; e-mail: sifessel@fcav.unesp.br

${ }^{3,4}$ Eng $^{\circ}$ Agr $^{\circ}$, Prof. Titular e Adjunto do Depto. de Produção Vegetal - FCAV/ UNESP.
}

que a semente precisa ser beneficiada e manipulada de forma adequada, caso contrário, os esforços anteriores para o desenvolvimento do material e as técnicas culturais para a produção das sementes podem ser perdidas.

Segundo Silveira \& Vieira (1982) a qualidade final da semente depende do cuidado em manter, durante o beneficiamento e o armazenamento, a qualidade obtida no campo, minimizando as injúrias que ocorrem durante o processamento, principalmente as injúrias mecânicas. A capacidade de uma semente de produzir uma planta normal pode ser 
reduzida ou anulada por injúrias mecânicas causadas durante o beneficiamento (Gregg et al., 1970).

A injúria mecânica é causada por choques e/ou abrasões das sementes contra superfícies duras ou contra outras sementes, resultando em sementes quebradas, trincadas, fragmentadas, arranhadas e inteiramente danificadas. Não só o aspecto físico da semente é atingido, pois sementes mecanicamente danificadas dificultam as operações de beneficiamento e apresentam menor germinação e vigor (Andrews, 1965 e Delouche, 1967). De acordo com Moore (1974), em produção mecanizada de sementes, as injúrias mecânicas são as maiores forças destrutivas que atuam na redução da qualidade fisiológica e sanitária das mesmas.

Para Delouche (1967) qualquer equipamento usado no manuseio é fonte potencial de danos mecânicos e de contaminação. Os transportadores, elevadores e outros equipamentos usados para movimentar sementes, desde a colheita, beneficiamento e embalagem, podem ter importante influência na qualidade da semente.

Reduções no vigor de sementes de milho foram observadas, após a operação de pré-limpeza (Albuquerque \& Priante-Filho, 1993). Resultados semelhantes também foram observados por Fessel et al., (1996) durante as etapas do beneficiamento de sementes de milho, como evidenciou os resultados da avaliação do dano mecânico, germinação, teste de tetrazólio e condutividade elétrica. Por outro lado Amaral et al. (1984) verificaram que a utilização de máquinas de ar e peneiras e mesa de gravidade eliminou materiais indesejáveis, aumentando a pureza física e sanitária de lotes de sementes de ervilha. Resultados semelhantes foram obtidos por Lollato \& Silva (1984) e Buitrago et al. (1991) quando constataram que sementes de feijão beneficiadas na mesa de gravidade apresentaram melhores qualidades físicas, fisiológicas e sanitárias.

Assim sendo, conduziu-se a presente pesquisa com o objetivo de avaliar a qualidade física, fisiológica e sanitária de sementes de milho durante as etapas de beneficiamento.

\section{MATERIAL E MÉTODOS}

O experimento foi conduzido no Laboratório de Análise de Sementes do Departamento de Produção Vegetal e no Laboratório de Patologia de Sementes do Departamento de Fitossanidade da Faculdade de Ciências Agrárias e Veterinárias - FCAV, UNESP, Câmpus de Jaboticabal.

As amostragens foram realizadas em 16 pontos do processamento do híbrido D766, safra 2001/2002 na unidade de beneficiamento de sementes da empresa Dow AgroScience. Os pontos de amostragens foram: (1) recepção, (2) pré-limpeza, (3) pós-mesa de gravidade - chato médio peneira 22 (PMG - CMP 22), (4) pós-mesa de gravidade - chato curto peneira 22 (PMG - CCP 22), (5) pós-mesa de gravidade redondo curto peneira 22 (PMG - RCP 22), (6) pós-mesa de gravidade - chato médio peneira 20 (PMG - CMP 20), (7) pós-mesa de gravidade - chato curto peneira 20 (PMG - CCP 20), (8) pós-mesa de gravidade - redondo curto peneira 20 (PMG - RCP 20), (9) pós-classificador - chato médio peneira 22 (PC - CMP22), (10) pós-classificador - chato curto peneira 22 (PC - CCP22), (11) pós-classificador - redondo curto peneira 22 (PC - RCP22), (12) pós-classificador - chato médio peneira 20 (PC - CMP20), (13) pós-classificador - chato curto peneira 20 (PC - CCP20), (14) pós-classificador - redondo curto peneira 20 (PC - RCP20), (15) pós-ensaque redondo curto peneira 22 (PE - RCP22) e (16) pós-ensaque redondo curto peneira 20 (PE - RCP20). Todas as sementes foram mantidas em câmara fria $\left(10^{\circ} \mathrm{C}\right.$ e umidade relativa de aproximadamente 50\%) durante o período de realização do trabalho.

As sementes foram avaliadas quanto ao: teor de água (TA) - determinado pelo método da estufa a $105 \pm 3^{\circ} \mathrm{C}$, por 24 horas, utilizando-se duas sub- amostras de 25 sementes por tratamento, conforme as Regras para Análise de Sementes (Brasil, 1992). Os resultados foram expressos em porcentagem; teste de germinação (TG) - realizado com quatro subamostras de 50 sementes por tratamento, semeadas em caixas plásticas $(26 \times 16 \times 10 \mathrm{~cm})$ contendo areia como substrato e mantidas em condições ambientais de laboratório $\left(25-30^{\circ} \mathrm{C}\right)$. A contagem final foi realizada no sétimo dia após a instalação do teste, considerando-se os critérios estabelecidos pelas Regras para Análise de Sementes (Brasil, 1992); primeira contagem da germinação - conduzido junto com o teste de germinação. Constituiu no registro das porcentagens de plântulas normais verificadas na primeira contagem do teste de germinação (4º dia) (Nakagawa, 1999); Índice de velocidade de germinação (IVG) - conduzido junto ao teste de germinação. As avaliações das plântulas foram realizadas diariamente, à mesma hora, a partir do dia em que surgiram as primeiras plântulas normais. As avaliações foram realizadas até o momento da última contagem (sétimo dia), estabelecida pelas Regras para Análise de Sementes (Brasil, 1992). Ao final do teste, o IVG foi calculado empregando se a fórmula de Maguire (1962); envelhecimento acelerado (EA) - conduzido segundo as recomendações contidas no manual de testes de vigor da ISTA (Hampton \& TeKrony, 1995) e também 
descrito por Marcos Filho, 1999. As sementes foram envelhecidas em caixas plásticas $(11 \times 11 \times 3,5 \mathrm{~cm})$ contendo 40mL de água deionizada, sobre uma tela, para evitar o contato das sementes com a água, colocadas em uma única camada. Depois do período de envelhecimento de 72 horas, por 45ㄷ (Hampton \& TeKrony, 1995 e Fessel et al., 2000), em câmara de envelhecimento acelerado, (modelo "water jackted”) foram determinados o teor de água das sementes e a germinação; teste de frio com solo (TF) - conduzido com quatro sub- amostras de 50 sementes por tratamento, semeadas em caixas plásticas $(26 \times 16 \times 10 \mathrm{~cm})$ contendo uma mistura de areia e terra (na proporção de 2:1). A adição de água foi realizada até atingir 70\% da capacidade de retenção do substrato. As caixas foram tampadas e colocadas em câmara fria $\left(10^{\circ} \mathrm{C}\right)$ por sete dias; posteriormente, retiradas, destampadas e mantidas em ambiente de laboratório por cinco dias, quando as plântulas normais foram contadas e os resultados expressos em porcentagem. Para a realização deste teste, seguiramse as recomendações da AOSA (1983) e Barros et al. (1999); condutividade elétrica (CE) - conduzido segundo recomendações da AOSA (1983) que constou do uso de quatro subamostras com 50 sementes por tratamento, pesadas com precisão de duas casas decimais e colocadas para embeber em copos plásticos (com capacidade de $200 \mathrm{~mL}$ ), contendo $75 \mathrm{~mL}$ de água deionizada. Os copos contendo as sementes foram mantidas em câmara de germinação, por 24 horas, à temperatura de $25^{\circ} \mathrm{C}$ (AOSA,1983; Loeffler et al., 1988 e Vieira \& Krzyzanowski, 1999). Decorrido o tempo de embebição, procedeu à leitura da condutividade elétrica, usando-se um condutivímetro 600 Analyser, com eletrodo com constante 1,0. Os resultados finais foram expressos em $\mu \mathrm{S} \mathrm{cm}^{-1} \mathrm{~g}^{-1}$; emergência das plântulas em campo (EC) - conduzido com quatro sub- amostras de 50 sementes por tratamento, em linhas de $2,5 \mathrm{~m}$ de comprimento e espaçamento de $0,50 \mathrm{~m}$ entre linhas e a profundidade de $5 \mathrm{~cm}$. A contagem das plântulas normais emergidas foi realizada aos 14 dias após a semeadura (Nakagawa, 1994); teste de sanidade - utilizou-se o método do papel de filtro com congelamento. As sementes foram incubadas em placas de Petri de 9cm de diâmetro, contendo, três folhas de papel de filtro umedecidas com água destilada. Utilizaram-se vinte placas por tratamento, com dez sementes em cada. Estas foram mantidas em ambiente controlado, com temperatura de $20 \pm 2^{\circ} \mathrm{C}$, em fotoperíodo de 12 horas de luz branca e 12 horas de escuro, seguindo-se um período de 24 horas à $-18^{\circ} \mathrm{C}$, e posteriormente retornado às condições iniciais de incubação por período de cinco dias. Após esse período de incubação a avaliação foi realizada em cada semente individualmente, sob microscópio estereoscópico, sendo a identificação dos fungos feita através de características morfológicas de seu crescimento sobre as sementes e, também pelo microscópio óptico, através de lâminas feitas do material contido nas sementes. Os resultados foram expressos em porcentagem sementes infectadas (Brasil, 1992); dano mecânico - conduzido segundo as recomendações de Oliveira et al. (1998). Constou - se do uso de quatro sub- amostras de 50 sementes para cada tratamento, colocadas para embeber na solução de Amaranth 0,1\% em copos plásticos (com capacidade de 200mL), cobrindo as sementes durante 2 minutos, agitando algumas vezes para facilitar a penetração da solução, e deixadas à temperatura ambiente. Decorrido o tempo de embebição, procedeu a lavagem das sementes em água corrente e avaliadas o número de sementes com danos, seguindo o critério de avaliação: Nota 0: sementes aparentemente sem danos; Nota 1: sementes com 10\% de área colorida, desde que distantes do embrião e ou leve absorção próximo ao ponto de inserção no sabugo; Nota 2: sementes com 10 a 40\% de área colorida, em qualquer ponto da semente, exceto no embrião; Nota 3: sementes acima de $40 \%$ da área do endosperma colorida ou com danos diretamente associados ao embrião. Os resultados finais foram expressos em porcentagem.

Análise estatística - os testes foram conduzidos no delineamento inteiramente casualizado, com quatro repetições por tratamento/teste, com exceção apenas para o teor de água em que usaram duas repetições por tratamento. A comparação das médias foi realizada usando-se o programa SAEG, teste de comparações múltiplas de Scott-Knott a 5\% de probabilidade. Para a análise dos dados da germinação, primeira contagem, envelhecimento acelerado, teste de frio, emergência das plântulas em campo e porcentagem de sementes contaminadas com fungos os dados foram transformados em arc sen $\sqrt{\mathrm{X} / 100}$, nas tabelas são apresentados às médias originais.

\section{RESULTADOS E DISCUSSÃO}

Na Tabela 1 estão apresentados os resultados do teor de água e do dano mecânico das sementes em cada etapa do beneficiamento. Pode-se observar que as sementes estavam com teor de água baixo, estando mais suscetível ao dano mecânico. Segundo Jijon \& Barros (1983) um dos fatores que influenciam a susceptibilidade das sementes ao dano mecânico é o seu grau de umidade. Estas, com baixos graus de umidade (10,60 a 11,76), são mais suscetíveis ao dano por 
TABELA 1. Teor de água inicial e após o envelhecimento acelerado e dano mecânico em sementes de milho durante as fases de beneficiamento.

\begin{tabular}{|c|c|c|c|c|c|c|}
\hline \multirow{3}{*}{$\begin{array}{c}\text { Etapas do } \\
\text { beneficiamento }\end{array}$} & \multicolumn{2}{|c|}{ Teor de água } & \multicolumn{4}{|c|}{ Dano mecânico } \\
\hline & Inicial & Após o EA & Nota 0 & Nota 1 & Nota 2 & Nota 3 \\
\hline & ............................ \% & 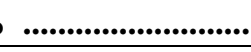 & .......................... & .................. & .................... & 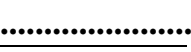 \\
\hline Recepção & $11,76 \mathrm{~A}$ & $22,36 \quad$ B & $93,5 \mathrm{~A}$ & $2,5 \quad \mathrm{~B}$ & $1,0 \mathrm{~A}$ & $3,0 \quad B$ \\
\hline Pré-limpeza & 10,60 В & $23,34 \mathrm{~A}$ & $92,5 \mathrm{~A}$ & $2,5 \mathrm{~B}$ & $0 \mathrm{~A}$ & $5,0 \quad \mathrm{~B}$ \\
\hline PMG - CMP 22 & $11,01 \mathrm{~B}$ & 22,54 B & 87,5 В & $5,5 \mathrm{~A}$ & $0,5 \mathrm{~A}$ & $6,5 \mathrm{~B}$ \\
\hline PMG - CCP 22 & $11,53 \mathrm{~A}$ & $23,56 \mathrm{~A}$ & $80,0 \quad C$ & $5,5 \mathrm{~A}$ & $1,5 \mathrm{~A}$ & $13,0 \mathrm{~A}$ \\
\hline PMG - RCP 22 & $11,45 \mathrm{~A}$ & 20,14 & 79,5 & $3,0 \quad \mathrm{~B}$ & $1,5 \mathrm{~A}$ & $16,0 \mathrm{~A}$ \\
\hline PMG - CMP 20 & 10,94 B & $24,11 \mathrm{~A}$ & $92,0 \mathrm{~A}$ & $2,5 \mathrm{~B}$ & $1,0 \mathrm{~A}$ & 4,5 В \\
\hline PMG - CCP 20 & $11,65 \mathrm{~A}$ & $23,53 \mathrm{~A}$ & 86,5 В & $3,0 \quad \mathrm{~B}$ & $0 \mathrm{~A}$ & $10,5 \mathrm{~A}$ \\
\hline PMG - RCP 20 & $11,27 \mathrm{~A}$ & $22,05 \quad$ B & 81,0 & $3,0 \quad \mathrm{~B}$ & $1,5 \mathrm{~A}$ & $14,5 \mathrm{~A}$ \\
\hline PC - CMP22 & $11,03 \quad$ B & $20,99 \quad \mathrm{C}$ & 88,0 B & $6,5 \mathrm{~A}$ & $0,5 \mathrm{~A}$ & $5,0 \quad \mathrm{~B}$ \\
\hline PC - CCP22 & $10,86 \mathrm{~B}$ & 22,03 B & 89,5 В & $1,5 \mathrm{~B}$ & $1,0 \mathrm{~A}$ & 8,0 B \\
\hline PC - RCP22 & $10,82 \quad$ B & 21,65 B & $80,5 \quad C$ & $3,5 \quad \mathrm{~B}$ & $1,5 \mathrm{~A}$ & $14,5 \mathrm{~A}$ \\
\hline PC - CMP20 & $11,98 \mathrm{~A}$ & $24,35 \mathrm{~A}$ & 85,5 & $6,0 \mathrm{~A}$ & $2,0 \mathrm{~A}$ & $6,5 \mathrm{~B}$ \\
\hline PC - ССР20 & 10,88 B & $22,38 \quad$ B & 84,0 & $4,5 \mathrm{~A}$ & $3,5 \mathrm{~A}$ & $8,0 \quad B$ \\
\hline PC - RCP20 & $11,24 \mathrm{~A}$ & $22,49 \quad$ B & 84,0 & $2,5 \mathrm{~B}$ & $1,5 \mathrm{~A}$ & $11,5 \mathrm{~A}$ \\
\hline PE - RCP22 & $11,43 \mathrm{~A}$ & $22,45 \quad$ B & 88,5 B & $2,5 \mathrm{~B}$ & $0 \mathrm{~A}$ & $9,0 \quad \mathrm{~B}$ \\
\hline PE - RCP20 & $11,33 \mathrm{~A}$ & $22,80 \quad \mathrm{~B}$ & 87,5 B & $1,5 \mathrm{~B}$ & $0 \mathrm{~A}$ & $11,0 \mathrm{~A}$ \\
\hline Teste F & $4,51^{* *}$ & $15,20^{* *}$ & $6,05^{* *}$ & $1,53 * *$ & $1,42^{* *}$ & $6,50 * *$ \\
\hline CV (\%) & 2,27 & 1,76 & 4,21 & 67,0 & 141,18 & 34,72 \\
\hline
\end{tabular}

${ }^{1}$ PMG - Pós-mesa gravitacional, CMP - Chato médio $n^{\circ}$ da peneira, CCP - Chato curto $n^{\circ}$ da peneira, RCP - Redondo curto $n^{\circ}$ da peneira, PC - Pós- classificador, PE - Pós- ensaque.

Médias seguidas pela mesma letra, maiúscula na coluna, não diferem entre si, pelo teste de Scott-Knott, a 5\%.

** Significativo a $1 \%$ de probabilidade

2 Nota 0: sementes aparentemente sem danos; Nota 1: sementes com 10\% de área colorida, desde que distantes do embrião e ou leve absorção próximo ao ponto de inserção no sabugo; Nota 2: sementes com 10 a 40\% de área colorida, em qualquer ponto da semente, exceto no embrião; Nota 3: sementes acima de $40 \%$ da área do endosperma colorida ou com danos diretamente associados ao embrião. Os resultados finais foram expressos em porcentagem.

quebramento. Segundo Carvalho \& Nakagawa (2000) a intensidade por quebramento começa a aumentar, à medida que o teor de água se reduz a valores inferiores a $12-14 \%$, e por amassamento aumenta em torno de $16-18 \%$, e dentro da faixa $12-14 \%$ a 16-18\% a intensidade de injúria mecânica seria mínima.

Ao longo das fases do beneficiamento ocorreu um aumento na porcentagem de dano mecânico, sendo importante destacar o efeito acumulativo dos danos no tegumento da semente, verificada pelo aumento da nota 3 . Os danos mecânicos nas sementes, além de apresentarem efeitos imediatos sobre sua qualidade, as predispõem à deterioração mais rápida, pelo aumento da respiração e da lixiviação de eletrólitos, proporcionando um maior percentual no número de plântulas fracas e anormais, maior susceptibilidade a microrganismos, maior sensibilidade aos fungicidas e redução no potencial de armazenamento (Bruggink et al., 1991 e Smith \& Berjak, 1995).

Considerando a porcentagem de germinação (Tabela 2), observou diferença significativa durante as fases de beneficiamento. Verificou-se que após as sementes passarem pela mesa de gravidade houve aumento na porcentagem de germinação, primeira contagem da germinação, no teste de frio, no envelhecimento acelerado e no índice de velocidade de germinação. Pode-se dizer que a mesa de gravidade interfere positivamente na qualidade fisiológica do lote, ao remover as sementes de menor densidade, quebradas e atacadas por patógenos.

Pelo teste de condutividade elétrica (Tabela 2) verificouse que a ocorrência de danos mecânicos propiciou aumento 
TABELA 2. Porcentagem de germinação, primeira contagem da germinação, índice de velocidade da germinação (IVG), teste de frio (TF), envelhecimento acelerado (EA), emergência em campo e condutividade elétrica (CE), obtidas durante as fases de beneficiamento.

\begin{tabular}{|c|c|c|c|c|c|c|c|c|}
\hline \multirow{4}{*}{$\begin{array}{c}\text { Etapas do } \\
\text { beneficiamento }^{1} \\
\text { Recepção }\end{array}$} & \multicolumn{8}{|c|}{ Vigor } \\
\hline & Germinação & $\begin{array}{c}1^{\circ} \text { contagem da } \\
\text { germinação }\end{array}$ & \multirow[t]{2}{*}{ IVG } & TF & \multicolumn{2}{|l|}{ EA } & \multirow{2}{*}{\multicolumn{2}{|c|}{$\begin{array}{c}\mathrm{CE} \\
\mu \mathrm{S} . \mathrm{cm}^{-1} \cdot \mathrm{g}^{-1}\end{array}$}} \\
\hline & \multicolumn{2}{|r|}{$\%$} & & \multicolumn{3}{|c|}{$\%$} & & \\
\hline & $92 \mathrm{~B}$ & $91 \mathrm{~A}$ & 23,52 B & $88 \mathrm{~A}$ & $58 \quad \mathrm{C}$ & 85 & 11,94 & $\mathrm{E}$ \\
\hline Pré-limpeza & $86 \mathrm{~B}$ & $85 \mathrm{~B}$ & $23,45 \quad B$ & $84 \mathrm{~A}$ & $84 \mathrm{~A}$ & $93 \mathrm{~A}$ & 11,86 & $\mathrm{E}$ \\
\hline PMG - CMP 22 & $97 \mathrm{~A}$ & $95 \mathrm{~A}$ & $26,90 \mathrm{~A}$ & $94 \mathrm{~A}$ & $93 \mathrm{~A}$ & $87 \mathrm{~A}$ & 9,81 & $\mathrm{E}$ \\
\hline PMG - CCP 22 & $96 \mathrm{~A}$ & $92 \mathrm{~A}$ & $28,33 \mathrm{~A}$ & $89 \mathrm{~A}$ & $89 \mathrm{~A}$ & $88 \mathrm{~A}$ & 12,34 & $\mathrm{E}$ \\
\hline PMG -RCP 22 & $95 \mathrm{~A}$ & $94 \mathrm{~A}$ & $27,33 \mathrm{~A}$ & $87 \mathrm{~A}$ & $70 \mathrm{~B}$ & $91 \mathrm{~A}$ & 11,00 & $\mathrm{E}$ \\
\hline PMG - CMP 20 & $97 \mathrm{~A}$ & $95 \mathrm{~A}$ & $29,18 \mathrm{~A}$ & $91 \mathrm{~A}$ & $87 \mathrm{~A}$ & 81 B & 11,60 & $\mathrm{E}$ \\
\hline PMG - CCP 20 & $93 \mathrm{~B}$ & $90 \mathrm{~A}$ & $27,88 \mathrm{~A}$ & $78 \mathrm{~B}$ & $83 \mathrm{~A}$ & $77 \quad \mathrm{C}$ & 15,94 & $\mathrm{C}$ \\
\hline PMG - RCP 20 & $90 \mathrm{~B}$ & $88 \mathrm{~A}$ & $26,78 \mathrm{~A}$ & $85 \mathrm{~A}$ & $88 \mathrm{~A}$ & $82 \mathrm{~B}$ & 18,18 & B \\
\hline PC - CMP22 & $87 \mathrm{~B}$ & $87 \mathrm{~B}$ & $26,84 \mathrm{~A}$ & $85 \mathrm{~A}$ & $73 \mathrm{~B}$ & $83 \mathrm{~B}$ & 13,28 & $\mathrm{D}$ \\
\hline PC - CCP22 & $91 \mathrm{~B}$ & $91 \mathrm{~A}$ & $27,71 \mathrm{~A}$ & $83 \mathrm{~A}$ & $73 \mathrm{~B}$ & $85 \mathrm{~B}$ & 15,12 & $\mathrm{C}$ \\
\hline PC - RCP22 & $95 \mathrm{~A}$ & $94 \mathrm{~A}$ & $26,69 \mathrm{~A}$ & $86 \mathrm{~A}$ & $89 \mathrm{~A}$ & $92 \mathrm{~A}$ & 13,47 & $\mathrm{D}$ \\
\hline PC - CMP20 & $94 \mathrm{~A}$ & $90 \mathrm{~A}$ & $27,01 \mathrm{~A}$ & $87 \mathrm{~A}$ & $75 \mathrm{~B}$ & $81 \mathrm{~B}$ & 12,83 & $\mathrm{D}$ \\
\hline PC - CСР20 & 88 B & $80 \mathrm{~B}$ & 23,89 B & $76 \mathrm{~B}$ & 74 B & $72 \quad \mathrm{C}$ & $21,62 \mathrm{~A}$ & \\
\hline PC - RCP20 & $83 \mathrm{~B}$ & $81 \mathrm{~B}$ & 24,13 B & $73 \mathrm{~B}$ & $70 \mathrm{~B}$ & $73 \mathrm{C}$ & $20,51 \mathrm{~A}$ & \\
\hline PE - RCP22 & $92 \mathrm{~B}$ & $89 \mathrm{~A}$ & $27,19 \mathrm{~A}$ & $89 \mathrm{~A}$ & $86 \mathrm{~A}$ & $83 \mathrm{~B}$ & 13,60 & $\mathrm{D}$ \\
\hline PE - RCP20 & $92 \mathrm{~B}$ & $90 \mathrm{~A}$ & $26,09 \mathrm{~A}$ & $86 \mathrm{~A}$ & $87 \mathrm{~A}$ & $80 \mathrm{~B}$ & $20,38 \mathrm{~A}$ & \\
\hline Teste F & $3,45^{* *}$ & $3,37 * *$ & $6,05^{* *}$ & $3,87 * *$ & $9,18^{* *}$ & $7,19 * *$ & 25,4 & 4 9** \\
\hline CV (\%) & 4,51 & 5,36 & 5,41 & 6,28 & 7,76 & 5,54 & 9,9 & \\
\hline
\end{tabular}

${ }^{1}$ PMG - Pós-mesa gravitacional, CMP - Chato médio $\mathrm{n}^{\circ}$ da peneira, CCP - Chato curto $\mathrm{n}^{\circ}$ da peneira, RCP - Redondo curto $\mathrm{n}^{\circ}$ da peneira, PC - Pósclassificador, PE - Pós- ensaque.

Médias seguidas pela mesma letra, maiúscula na coluna, não diferem entre si, pelo teste de Scott-Knott, a 5\%.

** Significativo a $1 \%$ de probabilidade.

de lixiviação de metabólitos, e conseqüentemente, a redução do vigor.

Nos testes de primeira contagem da germinação, teste de frio, envelhecimento acelerado, emergência em campo e índice de velocidade de germinação (Tabela 2) apresentaram comportamento similar, ou seja, ocorreram variações nas porcentagens de vigor das sementes em algumas fases do beneficiamento, sendo que a partir do pós-ensacamento o vigor aumentou, apresentando no final os mesmos níveis estatísticos do início do beneficiamento. Tal fato demonstra mais uma vez os efeitos benéficos do beneficiamento sobre a qualidade das sementes. Pela análise da Tabela 2, observou-se que o beneficiamento foi eficiente para melhorar a qualidade fisiológica deste lote.
Analisando os resultados do teste de sanidade (Tabela 3) verificou-se que houve redução na ocorrência dos fungos Fusarium sp. e Cladosporium sp. ao longo de cada etapa do beneficiamento, porém para Penicillium sp. e Cephalosporium sp. ocorreram aumentos progressivos, para Penicillium sp. este aumento ocorreu até o ponto de amostragem 6 (PMG CMP 20) a partir deste ponto sofreu oscilação mas acaba reduzindo, para Cephalosporium sp. este aumento foi até o ponto de amostragem 14 (PC - RCP 20), após a fase de pós-ensaque houve grande redução na incidência do patógeno. Na etapa de pós-ensaque pode-se observar baixa ocorrência destes fungos, com exceção do Fusarium sp., principalmente para sementes redondas - curta peneira 20, devido às sementes estarem tratadas quimicamente e este tratamento não controlou a 
TABELA 3. Porcentagens de sementes com incidência de fungos, em função da etapas de beneficiamento.

\begin{tabular}{|c|c|c|c|c|c|c|c|}
\hline \multirow{2}{*}{$\begin{array}{c}\text { Etapas do } \\
\text { beneficiamento }{ }^{1}\end{array}$} & Fusarium spp. & \multicolumn{2}{|c|}{ PenIcillium sp. } & \multicolumn{2}{|c|}{ Cladosporium sp. } & \multicolumn{2}{|c|}{ Cephalosporium sp. } \\
\hline & $\ldots$ & $\ldots \ldots \ldots \ldots$ & .................... & $\%$ & 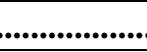 & 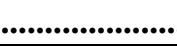 & ........................... \\
\hline Recepção & $30,0 \mathrm{~A}$ & 49,0 & C & $46,5 \mathrm{~A}$ & & 89,0 & B \\
\hline Pré-limpeza & $38,5 \mathrm{~A}$ & 59,5 & $\mathrm{C}$ & 13,5 & & 75,0 & $\mathrm{C}$ \\
\hline PMG - CMP 22 & $17,5 \quad \mathrm{~B}$ & 68,5 & & $36,5 \mathrm{~A}$ & & $86,0 \mathrm{I}$ & \\
\hline PMG - CCP 22 & 8,5 B & $94,5 \mathrm{~A}$ & & 9,0 & B & $96,5 \mathrm{~A}$ & \\
\hline PMG - RCP 22 & 17,5 В & 48,5 & C & 6,5 & $\mathrm{C}$ & $97,0 \mathrm{~A}$ & \\
\hline PMG - CMP 20 & $10,5 \quad \mathrm{~B}$ & $87,0 \mathrm{~A}$ & & $37,5 \mathrm{~A}$ & & $96,0 \mathrm{~A}$ & \\
\hline PMG - CCP 20 & 10,0 B & 17,0 & $\mathrm{E}$ & 3,5 & $\mathrm{D}$ & $98,0 \mathrm{~A}$ & \\
\hline PMG - RCP 20 & $11,0 \quad \mathrm{~B}$ & 49,0 & C & 3,5 & $\mathrm{D}$ & $91,0 \mathrm{~A}$ & \\
\hline PC - CMP 22 & 8,0 B & 32,0 & $\mathrm{D}$ & 4,0 & $\mathrm{D}$ & $95,0 \mathrm{~A}$ & \\
\hline PC - CCP 22 & 9,5 В & 50,0 & $\mathrm{C}$ & 13,5 & B & $93,0 \mathrm{~A}$ & \\
\hline PC - RCP 22 & $12,0 \quad \mathrm{~B}$ & 51,0 & $\mathrm{D}$ & 10,5 & B & $89,0 \mathrm{~A}$ & \\
\hline PC - CMP 20 & 9,0 B & 18,0 & $\mathrm{E}$ & 3,0 & $\mathrm{D}$ & $98,0 \mathrm{~A}$ & \\
\hline PC - ССР 20 & 7,5 В & 25,0 & $\mathrm{D}$ & 5,5 & $\mathrm{C}$ & $95,0 \mathrm{~A}$ & \\
\hline PC - RCP 20 & 4,5 B & 47,5 & $\mathrm{C}$ & 6,5 & $\mathrm{C}$ & $99,0 \mathrm{~A}$ & \\
\hline PE - RCP 22 & $10,0 \quad B$ & 0,5 & $\mathrm{~F}$ & 0 & E & 10,0 & $\mathrm{D}$ \\
\hline PE - RCP 20 & $31,5 \mathrm{~A}$ & 13,5 & E & 0 & E & 7,0 & E \\
\hline Teste F & $6,16^{* *}$ & $55,64 * *$ & & $23,05^{* *}$ & & $133,19 * *$ & \\
\hline CV (\%) & 25,15 & 16,69 & & 25,50 & & 4,61 & \\
\hline
\end{tabular}

${ }^{1}$ PMG - Pós- mesa gravitacional, CMP - Chato médio $\mathrm{n}^{\circ}$ da peneira, CCP - Chato curto $\mathrm{n}^{\circ}$ da peneira, RCP- Redondo curto $\mathrm{n}^{\circ}$ da peneira, PC - Pós-classificador, PE - Pós-ensaque.

Médias seguidas pela mesma letra, maiúscula na coluna, não diferem entre si, pelo teste de Scott-Knott, a 5\%.

** Significativo a $1 \%$ de probabilidade.

incidência. É importante ressaltar que a semente se constitui no meio mais eficiente de disseminação de patógenos. Observou-se, também a ocorrência não quantificada dos seguintes fungos: Aspergillus, Rhizopus, Molinea, Epicoccum, Alternaria e Curvularia.

\section{CONCLUSÃO}

Os danos mecânicos podem ocorrer a cada fase do beneficiamento e são cumulativos.

O beneficiamento de sementes de milho pode aprimorar a qualidade de um lote em termos de germinação, vigor e sanidade.

\section{REFERÊNCIAS}

ALBUQUERQUE, M.C.F.; PRIANTE-FILHO, N. Efeito da máquina de pré-limpeza na qualidade fisiológica de sementes de milho. In: CONGRESSO BRASILEIRO DE SEMENTES, 8, 1993, Campo Grande. Resumos... Informativo ABRATES, Curitiba, v.3, n3, p.36, 1993.

AMARAL, A.S.; BICCA, L.H.F.; WOBETO, L.A. Classificação de sementes de ervilha. Lavoura Arrozeira, Porto Alegre. v.348, p.3235, 1984.

ANDREWS, C. Mechanical injury on seeds. In: SHORT COURSE FOR SEEDSMAN, 1965, Mississipi. Proceedings... Mississipi State University, 1965. p.125-130.

AOSA - Association of Official Seed Analysts. Seed vigor testing handbook. East Lasing, 1983. 88p. (Contribution, 32).

BARROS, A.S.R.; DIAS, M.C.L.L.; CICERO, S.M.; KRZYZANOWSKI, F.C. Testes de frio. In: KRZYZANOWSKI, F.C.; VIEIRA, R.D.; FRANÇA NETO, J. de B. (Eds.) Vigor de Sementes: Conceitos e Testes. Londrina: ABRATES. 1999. p.5.1-5.15.

BRASIL. Ministério da Agricultura. Regras para análise de sementes. Brasília: SNAD/DNPV/ CLAV, 1992. 365p.

BUITRAGO, I.C.; VILLELA, F.; TILLMANN, M. A.A.; SILVA, J.B. Perdas e qualidade de sementes de feijão beneficiadas em máquinas de ventiladores e peneiras e mesa de gravidade. Revista Brasileira de Sementes, Brasília, v.13, n.2, p.99-104, 1991. 
BRUGGINK, H.; URARK, H.L.; DIJKEMA, M.H.G.F.; BEKENDAM, J. Some factors influencing electrolyte from maize (Zea mays L.) kernels. Seed Science Research, Zurich, v.1, n.1, p.15-20, 1991.

CARVALHO, N.M.; NAKAGAWA, J. Sementes: ciência, tecnologia e produção. 4.ed. Jaboticabal: FUNEP, 2000. 588p.

DELOUCHE, J.C. Mechanical damage to seed. In: SHORT COURSE FOR SEEDSMAN, 1967, Mississipi, Proceedings... Mississipi State University. p.69-71.

FESSEL, S.A.; VIEIRA, R.D.; FAGIOLI, M. Avaliação da qualidade fisiológica de sementes de três genótipos de milho, obtidas em quatro fases do processamento. In: VII Congresso de Iniciação Científica UNESP, 1996, Guaratinguetá. Anais... p.292.

FESSEL, S.A.; RODRIGUES, T.J.D.; FAGIOLI, M.; VIEIRA, R.D. Temperatura e período no teste de envelhecimento acelerado em sementes de milho. Revista Brasileira de Sementes, Brasília, v.22, n.2, p.163-170, 2000

GREGG, B.R.; LAW, A.G.; VIRDI, S.S.; BALIS, J.S. Seed processing. Mississipi: Mississipi State University, 1970. p.328-344.

HAMPTON, J.G.; TEKRONY, D.M. Handbook of vigour test methods. $3^{\text {rd }}$.ed., Zürich:ISTA, 1995.117p.

JIJON, A.V.; BARROS, A.C.S.A. Efeito dos danos mecânicos na semeadura sobre a qualidade de sementes de soja (Glycine Max (L.) Merril. Tecnologia de Sementes, Pelotas. V.6, n. 1/2, p. 3-22, 1983.

LOEFFLER, T.M.; TEKRONY, D.M.; EGLI, D.B. The bulk conductivity test as in indicator of soybean seed quality. J. Seed Technol., East Lansing, v. 12, n.1, p.37-53, 1988.

LOLLATO, M.A.; SILVA, W.R. Efeito da utilização da mesa de gravitacional na qualidade de sementes de feijão. Pesquisa Agropecuária Brasileira, Brasília. v.19, n.12, p.1483-1496, 1984.
MAGUIRE, J.D. Speed of germination and seedling emergence and vigour. Crop Science, Madison, v.2, n.2, p.176-177, 1962.

MARCOS FILHO, J. Teste de envelhecimento acelerado. In: In: KRZYZANOWSKI, F.C.; VIEIRA, R.D.; FRANÇA NETO, J. de B. (Eds.). Vigor de Sementes: Conceitos e Testes. Londrina: ABRATES. 1999. p.3.1-3.24.

MOORE, R.P. Effects of mechanical injuries on viability. In: ROBERTS, E.M. (Ed). Viability of seeds. London: Chapman and Hall. 1974. p.94-113.

NAKAGAWA, J. Testes de vigor baseados na avaliação das plântulas. In: VIEIRA, R.D.; CARVALHO, N.M. (ed.) Testes de vigor em sementes. Jaboticabal: FUNEP, 1994. p.49 -85.

NAKAGAWA, J. Testes de vigor baseados no desempenho das plântulas. In: KRZYZANOWSKI, F.C.; VIEIRA, R.D.; FRANÇA NETO, J. de B. (Eds.) Vigor de Sementes: Conceitos e Testes. Londrina: ABRATES. 1999. p.2.1-4.24.

OLIVEIRA, J.A.; CARVALHO, M.L.M.; VIEIRA, M.G.G.C.; SILVA, E.A.A. Utilização de corantes na verificação de incidência de danos mecânicos em sementes de milho. Revista Brasileira de Sementes., Brasília, v.20, n.2, p.363-366, 1998.

SILVEIRA, J.F.; VIEIRA, M.G.G.C. Beneficiamento de sementes. Informe Agropecuário, Belo Horizonte. v.8, n.9, p.50-56, 1982.

SMITH, M.T. \& BERJAK, P. Deteriorative changes associated with the loss of viability of stored desiccations of seed associated mycoflora during storage. In: JAIME, K.; GALILI, G. Seed development and germination. New York, Marcel Dekker Inc., 1995, p.701-746.

VIEIRA, R.D.; KRZYZANOWSKI, F.C. Teste de condutividade elétrica. In: KRZYZANOWSKI, F.C.; VIEIRA, R.D.; FRANÇA NETO, J. de B. (Eds.) Vigor de Sementes: Conceitos e Testes. Londrina: ABRATES. 1999. p.4.1-4.26. 